\title{
Sphenoid Sinus Mucocele - Rupture Causing Brainstem Inflammation and Stroke
}

\author{
Kevin Busche, William F. Morrish
}

\begin{abstract}
Background: Posterior rupture of a sphenoid sinus mucocele is a rare cause of brainstem injury. Methods: Case report. Results: A healthy young woman with a history of prior surgical excision of nasal polyps presented with a headache and evolving neurologic symptoms. The clinical presentation and imaging studies were consistent with a posterior rupture of a large sphenoid mucocele with subsequent inflammatory changes in the brainstem and evidence of brainstem stroke. Relatively rapid recovery coincided temporally with the resolution of local inflammation. Longer-term recovery continued over months, as expected following stroke. Conclusions: Posterior rupture of a sphenoid sinus mucocele may cause brainstem injury by multiple mechanisms.
\end{abstract}

RÉSUMÉ: Mucocèle du sinus sphénoïdal - Rupture provoquant une inflammation du tronc cérébral et un accident vasculaire cérébral. Contexte : La rupture postérieure d'un mucocèle du sinus sphénoïdal est une cause rare de lésion du tronc cérébral. Méthodes : Il s'agit d'une observation. Résultats : Une jeune femme en bonne santé, qui avait subi antérieurement une excision chirurgicale de polypes nasaux, a consulté pour une céphalée et des symptômes neurologiques progressifs. Le tableau clinique et l'imagerie étaient compatibles avec une rupture postérieure d'un gros mucocèle sphénoïdal ayant provoqué de l'inflammation et des signes d'accident vasculaire cérébral au niveau du tronc cérébral. La récupération s'est poursuivie pendant plusieurs mois, comme on peut s'y attendre après un accident vasculaire cérébral. Conclusions : La rupture postérieure d'un mucocèle du sinus sphénoïdal peut causer une lésion du tronc cérébral par plusieurs mécanismes.

Can. J. Neurol. Sci. 2008; 35: 381-385

A healthy 43-year-old right-hand dominant woman presented one evening with complaints of 48 hours of left-sided headache and facial pain, accompanied by a sense of left face numbness, which had developed 24 hours prior to presentation. The headache had come on gradually. It was described as throbbing with episodic worsening when the pain was described as sharp. There was no accompanying nausea, vomiting, phonophobia or photophobia. The patient had a history of chronic sinus congestion with associated headaches, but, the headache with this presentation was worse than those experienced previously. She was known to have a history of asthma and had had nasal polyps surgically removed multiple times. At the time of presentation, medications used by the patient included flonase, advair and airomir for sinus symptoms. The patient had a known allergy to non-steroidal anti-inflammatory drugs (NSAIDs).

She initially presented to an urgent care clinic; treatment with intravenous maxeran did improve the headache to a degree. She was transferred to the emergency room for further evaluation and imaging.
When seen in the emergency room she was afebrile and all vital signs were normal aside from a mildly elevated blood pressure. She was not in any distress and her neurological examination was described as being normal. Specifically noted was normal extraocular movements and light touch sensation on the face. There was no neck stiffness. There was found to be tenderness to palpation over the left side of the scalp in the parietal and occipital regions. 
Bloodwork did show a slightly elevated WBC (12.0) with a relative increase in neutrophils (10.0).

Initial imaging with a computed tomogram (CT) scan of the head revealed a large hyperdense sellar mass (Figure 1a). This was noted to extend through the skull base into the sphenoid sinus, with extension superiorly into the supra sellar region. There was noted to be calcification in the superior aspect of the mass. Opacification of the frontal, ethmoid and sphenoid sinuses was described. The initial impression was that this might represent a pituitary adenoma or meningioma, less likely to be invasive sinus disease. Further imaging with magnetic resonance image (MRI) was suggested, and the patient agreed to wait in the ER for this imaging to be completed.

Overnight, the patient remained stable. She complained to the nursing staff of some mild dizziness and numbness to the left face. She was still able to ambulate independently. Maxeran IV was given intermittently for mild headache.

The afternoon following her presentation, she developed acute ataxia when attempting to ambulate to the washroom. It was noted at this time that she was dysarthric and she seemed to
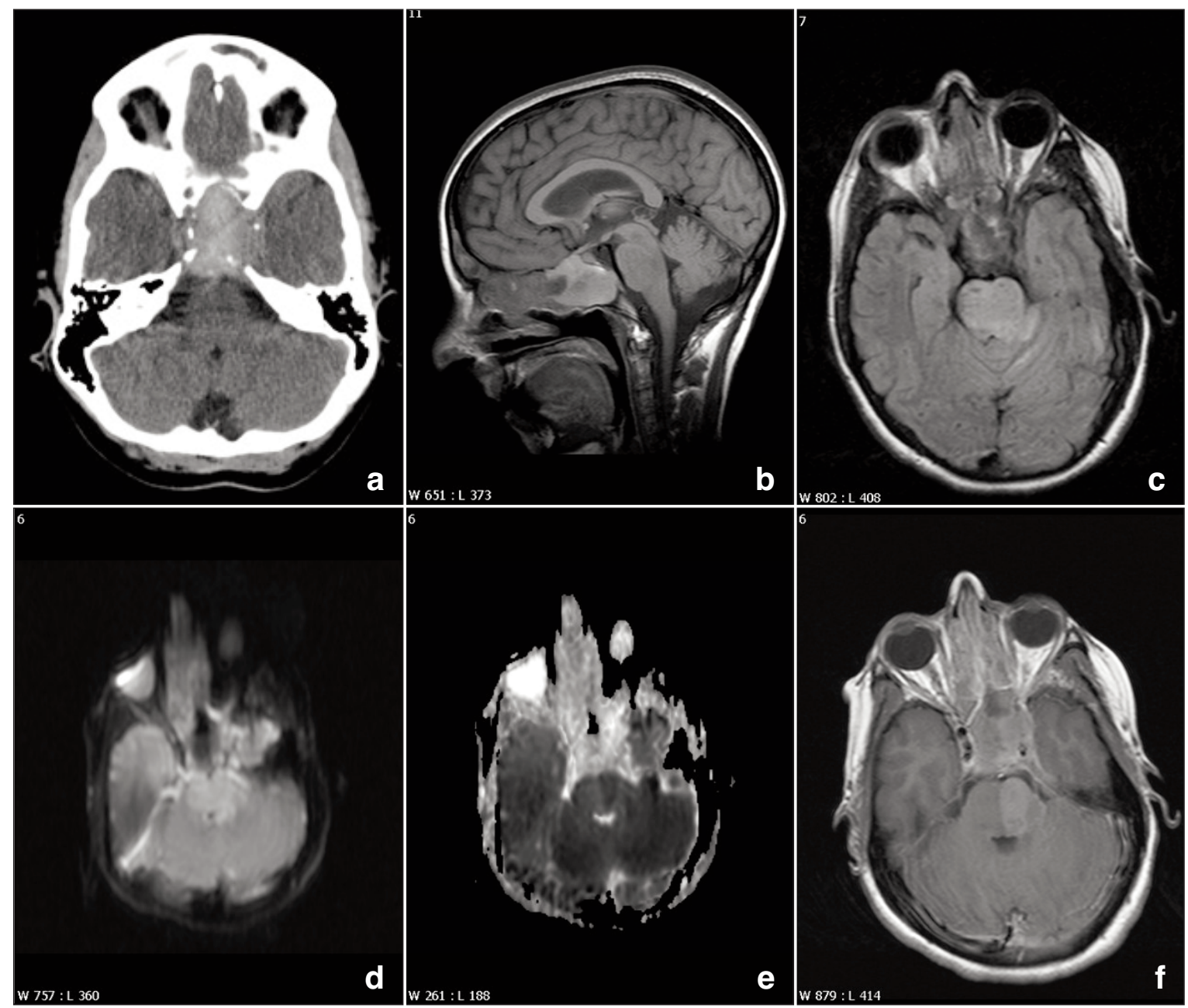

Figure 1: a. Single unenhanced axial CT image at the level of the sella demonstrates a large hyperdense destructive lesion. $b$. to $f$. Sagittal T1 (b) axial FLAIR (c), axial diffusion $(B=1000)(d)$, axial ADC map (e) and axial T1 post-Gadolinium enhanced images (f) from MRI performed the following day demonstrates a large sphenoid mucocele with increased T1 signal. This extends into the prepontine cistern (a) into the sella displacing the pituitary gland superiorly and posteriorly $(a)$ and into the left cavernous sinus $(f)$. FLAIR imaging and diffusion imaging demonstrate increased signal throughout the pons and in the left cerebellum/left middle cerebellar peduncle. ADC map shows a well demarcated area of decreased signal in the left side of the pons representing restricted diffusion/ischemic change. There is no restricted diffusion in the right pons consistent with edema. There is a possible small area of restricted diffusion in the left middle cerebellar peduncle representing a small additional area of acute ischemic change. Changes in the left cerebellum and left middle cerebellar peduncle are predominantly edema. Enhancement of the left pons $(f)$ is consistent with blood brain barrier breakdown in ischemic parenchyma. The basilar artery remains patent as a flow void is demonstrated (a). Well demarcated ischemic change in the left pons is consistent with a basilar perforator infarct. 
be cognitively slowed. She continued to describe left face numbness, with a new complaint of right hand numbness. The patient became nauseated and started vomiting. She complained of double vision.

Magnetic resonance imaging was completed urgently and compared to the CT of the previous evening. The MRI (Figure 1b-f) revealed a large expansile mass within the sphenoid sinus. Bony erosion was seen. There was extension of the abnormal signal into the prepontine cistern and the left cavernous sinus. There was noted to be diffuse signal abnormality within the pons, greater on the left than the right, extending into the left middle cerebellar peduncle, both cerebral peduncles extending superiorly to the left basal ganglia region. There was noted to be restricted diffusion in the left pons consistent with acute infarction. There was gadolinium enhancement seen within the left pons and local leptomeningeal enhancement as well. It was felt that the imaging changes demonstrated a sphenoid sinus mucocele extending posteriorly into the prepontine cistern and left cavernous sinus. It was thought that the territory of the apparent infarction was consistent with involvement of local pontine perforating arteries, presumably secondary to chemical or infectious vasculitis.

Following the MRI, telephone consultation with the neurosurgery service was obtained and it was not felt that there was any role for neurosurgical intervention. Neurology consultation was obtained. At the time of the initial neurology assessment (24 hours after the patient initially presented) she was afebrile with stable vital signs but appeared unwell and in distress. She was still alert, although mildly confused, suggesting that she would like to go home and return for treatment at a later time. She was noted to be dysarthric. Right ptosis was present. There was left gaze-evoked nystagmus. There was reduced left facial sensation to light touch. Motor tone, was mildly increased in both legs. Motor power was normal throughout. The reflexes were diffusely brisk and both toes were upgoing. Coordination of the upper limbs was poor bilaterally in the arms, worse on the left than the right. There was marked gait ataxia.

The patient was started on ceftriaxone, flagyl and dexamethasone. Otolaryngology consultation was obtained and the patient was taken to the operating room. The ethmoid and sphenoid sinuses were explored and evacuated, with large amount of mucopurulent discharge suctioned out of the sphenoid sinus. No cerebrospinal fluid (CSF) was encountered. The patient was observed in the intensive care unit following this procedure.

The next morning, the patient showed worsening neurologic function. She complained of horizontal diplopia. She was drowsy and her dysarthria had worsened. There was limitation of rightward gaze with upbeating nystagmus at rest with superimposed gaze-evoked nystagmus in all directions. There was reduced power (4/5) in the right arm and leg and more appendicular ataxia in the upper extremities.

An infectious disease consultation was obtained. It was felt that it was more likely to be a chemical meningitis than infection, given the lack of a toxic presentation, the absence of organisms or neutrophils in the material evacuated from the sphenoid sinus and the maintenance of an afebrile state throughout both the subacute and acute portions of the illness. Nevertheless, vancomycin was added to the ceftriaxone and flagyl regimen.

Re-examination later that same day showed an improved level of consciousness with the patient awake and more alert; her dysarthria, however, had worsened to the point that her speech was almost unintelligible. She showed mild bilateral ptosis, worse on the right with some restricted upgaze on extraocular movements. There was still decreased light touch to the left face; there also now appeared to be some reduction of the right nasolabial fold, although facial movements otherwise appeared normal. Her motor tone was diffusely increased. Right-sided weakness was diffusely $3 / 5$ in the arm and leg. Reflexes were
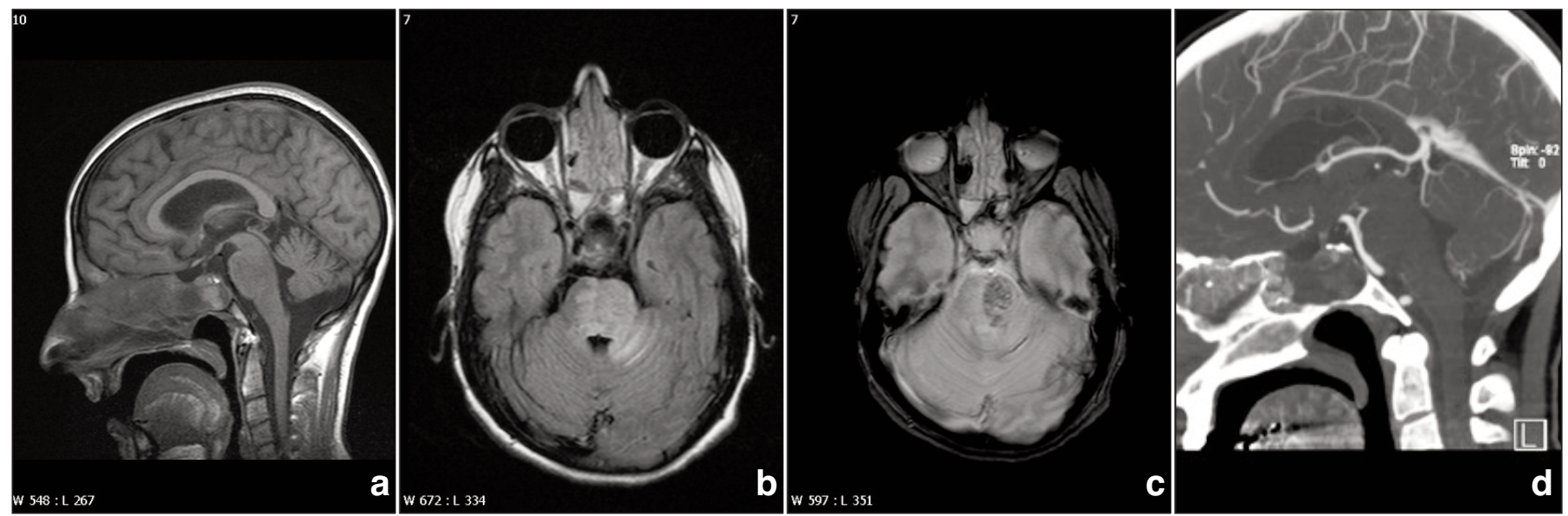

Figure 2: Sagittal T1 (a), axial FLAIR (b), axial gradient echo (c) 23 days post drainage of sphenoid mucocele and antibiotic therapy. Mucocele markedly diminished in size (a). Changes in left cavernous sinus have regressed (b). Evolving infarct in left pons on FLAIR imaging demonstrates extensive petechial hemorrhage on gradient echo imaging(c). A small amount of hemorrhage is also seen in the left cerebellum (c). Persistent edema in right pons and left cerebellum $(b)$. Sagittal MIP image from a CTA $(d)$ seven days post presentation demonstrates persistent patency of the basilar artery. 

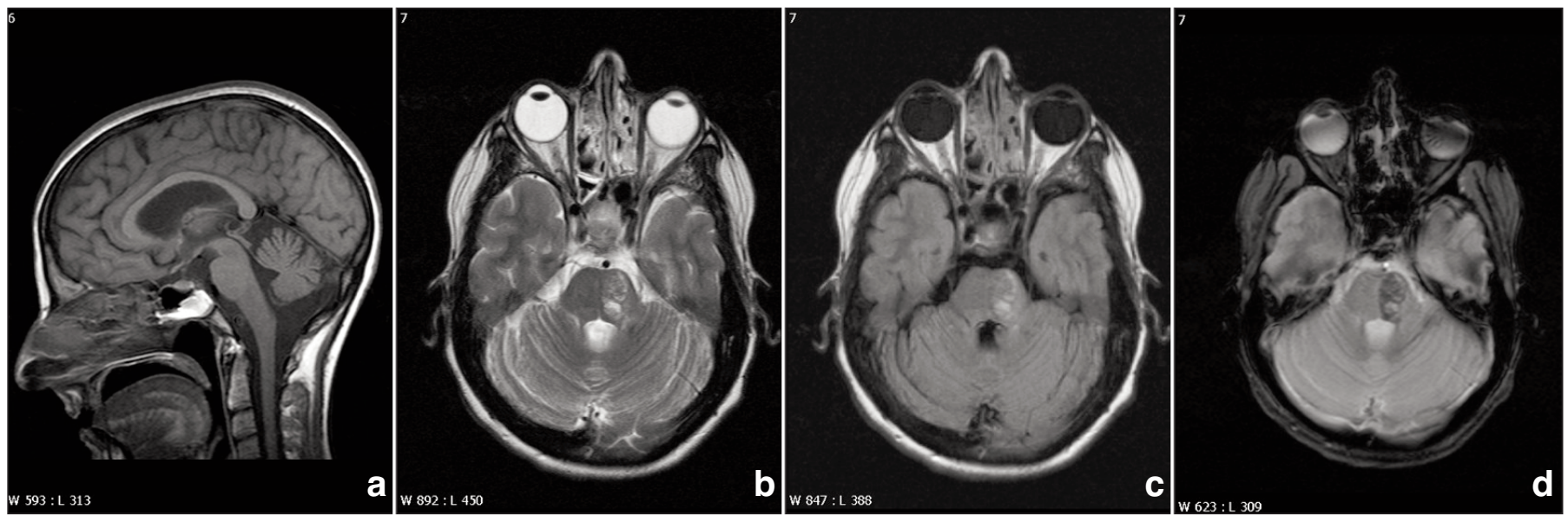

Figure 3: Sagittal T1 (a), axial T2 (b), axial FLAIR (c), axial gradient echo (d) from MRI nine months post initial studies. Mucocele has further regressed. There is persistent soft tissue in the sphenoid sinus, hyperintense on $T 1$, likely proteinaceous in nature. Left pontine infarct has evolved and the associated mass effect has resolved $(b, c)$. Hemosiderin deposition in left pons persist as does a small amount of hemosiderin deposition in the left cerebellum $(d)$. Edema in right pons and left cerebellum has resolved $(b, c)$.

brisk throughout without pathologic spread and were still symmetric; the right toe was upgoing. Right sided coordination was not well assessed because of weakness but the left arm and leg showed marked incoordination. There was reduced light touch sensation in the right arm while the right leg was normal. The exam was largely unchanged the following day. Plavix was added to the drug regimen.

The patient was subsequently transferred out of the ICU to the inpatient neurology service. Further infectious disease consultation led to the conversion of the antibiotic regimen to ceftazadime, metronidazole, cloxacillin, vancomycin and rifampin. The antibiotics were converted to meropenem approximately one week later.

All blood cultures were negative. The final cultures of the sphenoid sinus tissue revealed few Staphylococcus aureus and scant coagulase negative staphylococci and Propionibacterium acnes. No fungal elements or yeast were seen in the sphenoid samples.

Repeat MRI imaging during the hospital stay showed considerable improvement in the inflammation in and around the pons with evolutionary changes consistent with the known left pontine infarction (Figure $2 \mathrm{a}, \mathrm{b}, \mathrm{c}$ ). The $\mathrm{CT}$ angiogram (Figure 2d) did not show significant abnormality. Extensive pansinusitus changes were seen.

During her two-week stay on the neurology unit, she showed remarkable recovery. There was rapid improvement of diplopia and gait ataxia; she was able to walk with stand-by assistance and had no complaints of double vision at the time of transfer to the rehabilitation unit.

She was transferred to the inpatient neuro-rehabilitation unit for a further six weeks. Her decadron was tapered and discontinued. By the time of discharge, she was noted to have achieved independence with all activities of daily living. There was noted to still be some mild dysarthria and some difficulties with right-sided weakness as well as some impulsivity. Her meropenem was discontinued after a six-week course; the plavix was also discontinued at the time of discharge.

Neurologic examination as an outpatient two days after discharge showed normal cognition. There was still mild dysarthria. Extraocular movements were normal and there was no nystagmus. The motor exam showed clonus at both ankles, sustained on the right and five beats on the left. There was spasticity throughout the arm and leg. Right arm power was $4 / 5$ for shoulder abduction, but, otherwise was normal. Power was diffusely $4 / 5$ in the right leg. Reflexes were all $3+$ on the right and in the left leg; left arm reflexes were $2+$. Toes were downgoing bilaterally. Coordination was mildly impaired in the right arm and leg and the gait was mildly ataxic. Despite ongoing complaints of parathesia in the right hand and foot, all sensory testing was normal.

At the time of further follow-up nine months later, the patient had returned to an exercise program of swimming, weight-lifting and running (for up to 20 minutes on a treadmill). She had returned to golfing and was planning to return to her work as an accountant. She was aware of some mild stiffness and weakness on the right side but otherwise felt well.

On examination there was some left lateropulsion on upward gaze. There were three beats of clonus at the right ankle and a spastic catch in the right arm. The right arm movements were a bit more slow and deliberate than the right but the motor power was normal throughout. Reflexes were $3+$ bilaterally at the knees and $2+$ elsewhere. The gait was minimally spastic but not ataxic. Otherwise the neurological exam was normal.

Repeat MRI (Figure 3 a,b,c,d) showed expected evolution of the pontine stroke with changes consistent with Wallerian degeneration. The inflammatory changes in the brainstem had resolved. There was still marked thickening of the mucosa of the sinuses. 
It appears that this patient experienced the posterior rupture of a sphenoid mucocele into the prepontine cistern with secondary meningitis and brainstem encephalitis. As a result of this, there was apparent local vasculitis affecting pontine perforating branches causing ischemic stroke in the left pons. Whether the inflammatory process was chemically induced or due to infection is still unclear; there was no convincing evidence of intracranial infection, although CSF studies were not obtained in this case.

Presumably, her initial rapid recovery was related to resolution of the inflammatory component of the brainstem injury. The slower improvement would be more in keeping with the timeline expected for improvement following stroke in a young person. The improvement seen on the imaging studies parallels the clinical improvement seen.

This appears to be the only case in the literature of a posterior circulation infarction secondary to local extension of a sphenoid mucocele posteriorly.

There is a case report of stroke in a ten-year-old girl following acute pansinusitis attributed to direct chemical or inflammatoryinduced vasospasm at the level of the cavernous carotid due to the proximity of the sphenoid sinusitis. ${ }^{1}$ In this case the stroke was in the right lentiform nucleus and internal capsule. CSF examination showed no evidence of intracranial infection; the cultured material that was surgically evacuated from the sphenoid sinus grew non-haemolytic Streptococcus.
Four cases of anterior circulation stroke associated with pansinusitis (all cases including involvement of the sphenoid sinus) were reported by Perez Barreto et al. ${ }^{2}$ In these cases there was stenosis or occlusion of the internal carotid artery demonstrable on imaging. This was speculated to be due to exposure of the internal carotid artery to inflammatory mediators causing perivascular inflammation and thrombosis, occasioned by the proximity of the cavernous carotid to the sphenoid sinus. It was noted that interleukin-1, a proinflammatory and procoagulant cytokine, can be found in the sinus mucosa in sinusitis.

A case report ${ }^{3}$ also outlines the story of a patient with multiple cranial nerve palsies and venous pontocerebellar infarction due to cavernous sinus thrombosis secondary to sphenoid sinusitis. In this case there was associated periorbital edema, chemosis and exophthalmos, features absent in the patient described in our report.

\section{REFERENCES}

1. Rochat P, von Buchwald C, Wagner A. Sinusitis and ischemic Stroke. Rhinology. 2001; 39: 173-5.

2. Perez Barreto M, Sahai S, Ameriso S, Ahmadi J, Rice D, Fisher M. Sinusitus and carotid artery stroke. Ann Otal Rhinol Laryngol. 2000; 109: 227-30.

3. Macdonald RL, Findlay JM, Tator CH. Sphenoethmoidal sinusitis complicated by cavernous sinus thrombosis and ponto-cerebellar infarction. Can J Neurosci. 1988; 15: 310-13. 